\title{
Modificação Química de Poli(Tereftalato de Etileno) Pós-Consumo por Reação com Ácido Sulfúrico: Estrutura e Propriedades
}

\author{
Janaína G. Alonso, Elisabeth C. Molina, Edvani C. Muniz, Adley F. Rubira \\ Departamento de Química, UEM \\ Gizilene M. de Carvalho \\ Universidade Norte do Paraná, UNOPAR
}

\begin{abstract}
Resumo: PET pós-consumo foi modificado quimicamente através de reação com ácido sulfúrico por diferentes tempos. O material modificado foi caracterizado por DSC e FTIR-PAS e XPS. Verificou-se que a intensidade relativa do isômero trans diminui enquanto do isômero gauche aumenta para tempos de reação entre 0 e 60 minutos. Após 60 minutos de reação observou-se a presença de endotermas múltiplas que podem ser relacionadas com o desenvolvimento de estruturas intermediárias porque as intensidades relativas dos isômeros trans e gauche não apresentam variação significativa. $\mathrm{O}$ valor determinado de capacidade de troca iônica do PET pós-consumo modificado (PETS-pc) é comparável com os valores de resinas ácidas comerciais e não é afetado no intervalo de tempo de reação utilizado. Os valores da capacidade máxima de adsorção, qm, e do parâmetro de afinidade entre o adsorvente e o adsorvato, $\mathrm{K},(111,18 \mathrm{ppm}$ e 531,91 mg de corante/100g de adsorvente, respectivamente) para a adsorção do corante Remazol vermelho na amostra quimicamente modificada por 30 minutos foram calculados a partir da isoterma de adsorção de Langmuir. Os resultados indicam que esse material possui características adsorventes, podendo vir a ser utilizado para tratamento de efluente de indústria de tingimento têxtil.
\end{abstract}

Palavras-chave: PET, modificação química, reciclagem, capacidade de troca iônica, adsorção.

\section{Chemical Modification of Post Consumer Poly(Ethylene Terephthalate) by Sulfuric Acid Reaction: Structure and Properties}

Abstract: Chemical modification of post consumer PET was carried out by reaction with sulfuric acid. The modified material was characterized by DSC, XPS and FTIR-PAS measurements. The relative intensity of trans isomer increases while it decreases for gauche isomer for reaction times from 0 up to 60 min. After 60 minutes of reaction multiple endotherms were observed, which may be related to the development of an intermediated structure, since the relative intensities of the trans and gauche isomers do not change. The ionic exchange capacity of the modified post consumer poly(ethylene terephthalate), PETS-pc, is comparable to commercial acid resins and is not affected by the reaction time. The maximum adsorption capacity, $\mathrm{q}_{\mathrm{m}}$ and the affinity parameter, $\mathrm{K},(111.18 \mathrm{ppm}$ and $531.91 \mathrm{mg}$ dye $/ 100 \mathrm{~g}$ adsorvent, respectively) due to the adsorption of Remazol Red were calculated for PET samples chemically modified during 30 minutes from the Langmuir adsorption isotherms. The results indicate that PETS-pc has excellent adsorvent capacity, and can be applied to treatment of textile dye effluents.

Keywords: PET, chemical modification, recycling, ion exchange capacity, adsorption.

\section{Introdução}

O poli(tereftalato de etileno) (PET) possui uma ampla utilização devido às suas excelentes propriedades ${ }^{[1]}$. Entre as fibras sintéticas, o PET apresenta uma estrutura cristalina compacta com boas propriedades mecânicas, dielétricas e ópticas, resistentes a vários solventes, ácidos e meios alcalinos ${ }^{[2,3]}$. Entre os vários usos industriais do PET podemos destacar o seu uso na área automotiva, eletro-eletrônica e de embalagens para gêneros alimentí- $\operatorname{cios}^{[4]}$. Sua aplicação como embalagem tem gerado uma grande quantidade de rejeitos urbanos, despertando grande interesse no reaproveitamento desse material a partir de processos de reciclagem. A reciclagem de polímero é uma questão significativa devido a vários aspectos: necessidade de diminuir a quantidade de rejeito urbano, conservar reservas de energia não renováveis e agregar valor social e ecológico a produtos, valorizando a qualidade de vida e estendendo uma nova oportunidade de recuperação e equilíbrio ao meio-ambiente ${ }^{[5]}$.

Autor para Correspondência: Gizilene M. de Carvalho, Departamento de Química, Universidade Estadual de Maringá, Av. Colombo 5790, CEP: 87020-900, Maringá, PR. E-mail: gmcarvalho@uem.br 
A reciclagem primária, também chamada de reciclagem pré-consumo, é efetuada na própria indústria geradora do resíduo, ou por outras empresas transformadoras, com materiais termoplásticos provenientes de resíduos industriais, que são limpos e de fácil identificação, não contaminados por impurezas. Um outro método de reciclagem, a reciclagem secundária, consiste na coleta, granulação e reprocessamento (térmico e/ou mecânico) do PET com a finalidade de obter diferentes produtos. A desvantagem deste método é que o material reciclado apresenta propriedades térmicas e mecânicas inferiores àquelas do polímero virgem. Esse problema pode ser contornado pela adição de aditivos ou misturando o material reciclado a um outro polímero (blenda) com o objetivo de melhorar alguma propriedade específica. A reciclagem química $^{[6,7]}$ ou terciária consiste na despolimerização das cadeias do polímero pela ação de solventes (clivagem solvolítica). Neste processo podem-se obter oligômeros de diferentes massas molares ou, quando a reação de despolimerização é levada ao limite, monômeros, que podem ser purificados possibilitando o retorno às matérias-primas originais, usadas novamente para a fabricação do mesmo produto. Quando a reação de despolimerização não é levada ao limite, a estrutura do material obtida na despolimerização pode ser variada em função do tempo e das condições de reação. A determinação da estrutura e das propriedades do material quimicamente modificado é fundamental para definir as suas diferentes possibilidades de aplicação.

O objetivo deste trabalho é modificar quimicamente o poli(tereftalato de etileno) pós-consumo, PETpc, através de reação em ácido sulfúrico, oferecendo uma nova alternativa de reciclagem para esse material. O material obtido foi caracterizado por DSC, FTIR-PAS, XPS e capacidade de troca iônica. Foi avaliada a utilização do material pós-consumo quimicamente modificado como adsorvente de corantes utilizados em indústrias de tingimento de têxteis.

\section{Experimental}

\section{Modificação Química.}

O PET-pc utilizado nesse trabalho foi cedido pela Plaspet, Maringá-PR, na forma de flocos. Os flocos foram lavados e secos a $60{ }^{\circ} \mathrm{C}$ por $24 \mathrm{~h}$. Aos flocos foi adicionado ácido sulfúrico comercial (96\%), na proporção de uma parte em massa de polímero para 10 partes em volume de ácido. A mistura reacional foi mantida sob agitação em temperatura ambiente por diferentes tempos entre 10 e 120 minutos. Após os tempos determinados de reação o material foi precipitado em água destilada e filtrado para remoção do excesso de ácido. A amostra de PET não modificada será designada por PETpc e as amostras quimicamente modificadas serão designadas por PETSpcx, onde x indica o tempo de reação do PETpc com ácido sulfúrico, em minutos.

\section{Caracterização do PETpc e doPETSpc.}

Espectroscopia na região do infravermelho, modo foto acústica (FTIR-PAS), foi realizada em um espectrofotômetro
BOMEN, modelo MB-100. Foram analisadas as bandas em $898 \mathrm{~cm}^{-1} \mathrm{e}$ em $973 \mathrm{~cm}^{-1}$, referente aos isômeros trans e gauche, respectivamente ${ }^{[8]}$. O comportamento térmico do material foi analisado por calorimetria exploratória diferencial (DSC), em um calorímetro Shimadzu, modelo 150 , com relação à posição e ao número de endotermas presentes. O mapeamento dos diferentes átomos na superfície da amostra de PETpc e de PETSpc foi analisado por espectroscopia de fotoelétrons num equipamento Perkin-Elmer Phi Model 5300.

\section{Determinação da capacidade de troca.}

Amostras de PETpc ou PETSpc foram colocadas em contato com a solução padronizada de $\mathrm{NaOH} 0,1 \mathrm{~mol} / \mathrm{L}$ durante 24 hs. Uma alíquota da solução sobrenadante foi tomada e titulada com solução padrão de $\mathrm{HCl} 0,1 \mathrm{~mol} / \mathrm{L}$ para se determinar a capacidade de toca iônica do material ${ }^{[9]}$.

\section{Determinação de Isotermas de Adsorção do Corante Remazol vermelho $R B$}

\section{i) Determinação da razão ideal massa de PETSpc/vo- lume de solução}

Soluções contendo $2,5 \%, 5 \%, 10 \%$ e $15 \%$ de massa de PETSpc/volume solução foram colocadas em contato, sob agitação constante, com solução de corante Remazol Vermelho RB 133\% (C.I. Reactive Red 198) 70 ppm a $30^{\circ} \mathrm{C}$ durante 24 horas sem controle de $\mathrm{pH}$. Após esse período, a suspensão foi filtrada e a solução foi analisada por espectrofotometria UV-VIS a $514 \mathrm{~nm}$.

\section{ii) Determinação da isoterma de adsorção}

A isoterma de adsorção foi obtida através do contato de quantidades fixas do adsorvente com soluções de corante em diferentes concentrações (5 a $5000 \mathrm{ppm}$ ) sem controle de $\mathrm{pH}$. Os ensaios foram realizados a $30^{\circ} \mathrm{C}$, utilizando a concentração ideal de adsorvente determinada como descrito na seção anterior. Após 24 horas sob agitação a suspensão foi filtrada e a solução analisada por espectrofotometria UV-VIS a $514 \mathrm{~nm}^{[10]}$.

\section{Resultados e Discussão}

O estado químico dos elementos componentes das amostras de PETpc e PETSpc submetidas a diferentes tempos de reação com ácido sulfúrico foi analisado por XPS. Os espectros foram resolvidos para a obtenção dos fotopicos dos elementos presentes e as suas concentrações relativas. Para o PETpc o fotopico do carbono 1s $(\mathrm{C}(1 \mathrm{~s}))$ pode ser resolvido em três sinais: (a) 284,6 $\pm 0,1 \mathrm{eV}$ para o carbono do anel aromático; (b) $286,2 \pm 0,1 \mathrm{eV}$ para o carbono éter e (c) $288,7 \pm 0,1 \mathrm{eV}$ para o carbono carbonílico (Figura 1a). Baseado na unidade repetitiva do PET a área relativa teórica desses picos é de 60:20:20, mas a razão encontrada foi de 65:18:17. O fotopico do oxigênio $1 \mathrm{~s}(\mathrm{O}(1 \mathrm{~s}))$ pode ser resolvido em 2 sinais: (a) $531,6 \pm 0,1 \mathrm{eV}$ para o oxigênio ácido e (b) 533,3 $\pm 0,1 \mathrm{eV}$ para o oxigênio éter (Figura $1 b$ ). A área relativa teórica para o O (1s), baseada na unidade repetitiva do PET é de 1:1, e a 

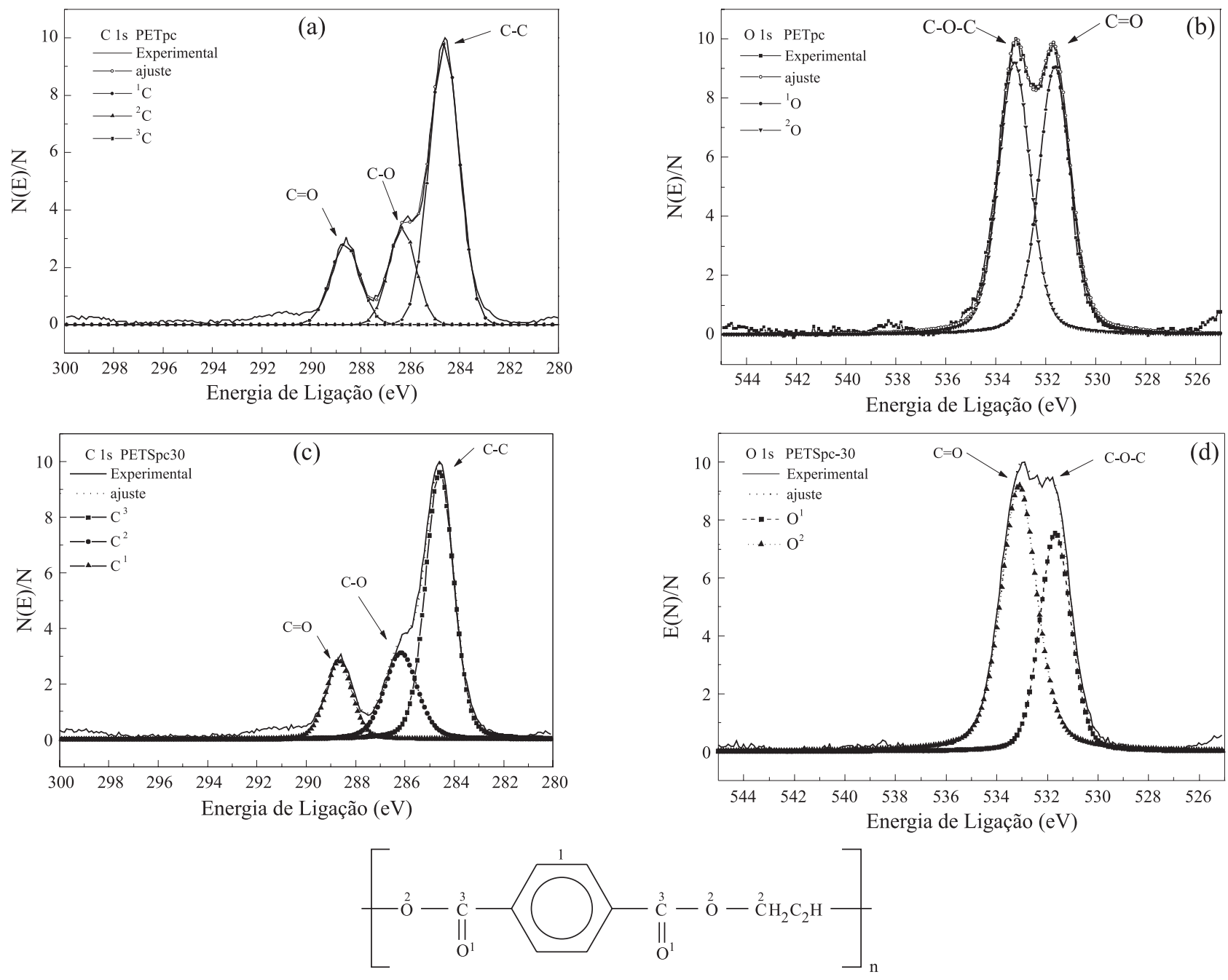

Figura 1. Fotopicos da amostra de PETpc (a) C (1s) e (b) O (1s) e fotopicos da amostra PETSpc30 (c) C (1s) e (d) O (1s).

Tabela 1. Valores de energia de ligação e concentração atômica relativa das espécies de carbono (1s) e oxigênio(1s) no PETpc e PETSpc.

\begin{tabular}{|c|c|c|c|c|c|c|c|c|c|c|c|}
\hline \multirow{2}{*}{\multicolumn{2}{|c|}{ Elemento }} & \multicolumn{2}{|c|}{ PETpc } & \multicolumn{2}{|c|}{ PETSpc30 } & \multicolumn{2}{|c|}{ РETSpc60 } & \multicolumn{2}{|c|}{ PETSpc90 } & \multicolumn{2}{|c|}{ PETSpc120 } \\
\hline & & $\begin{array}{l}\text { Pico } \\
(e V)\end{array}$ & $\begin{array}{c}\% \\
\text { atômica }\end{array}$ & $\begin{array}{l}\text { Pico } \\
(e V)\end{array}$ & $\begin{array}{c}\% \\
\text { atômica }\end{array}$ & $\begin{array}{l}\text { Pico } \\
(\mathrm{eV})\end{array}$ & $\begin{array}{c}\% \\
\text { atômica }\end{array}$ & $\begin{array}{l}\text { Pico } \\
(\mathrm{eV})\end{array}$ & $\begin{array}{c}\% \\
\text { atômica }\end{array}$ & $\begin{array}{l}\text { Pico } \\
(\mathrm{eV})\end{array}$ & $\begin{array}{c}\% \\
\text { atômica }\end{array}$ \\
\hline \multirow{3}{*}{$\mathrm{C}(1 \mathrm{~s})$} & 1 & 284,6 & 64,7 & 284,5 & 60,2 & 284,5 & 59,1 & 284,4 & 60,2 & 284,6 & 59,2 \\
\hline & 2 & 286,2 & 18,1 & 286,2 & 23,4 & 286,2 & 25,1 & 286,1 & 25,8 & 286,2 & 28,0 \\
\hline & 3 & 288,7 & 16,8 & 288,6 & 16,4 & 288,7 & 15,8 & 288,5 & 14,0 & 288,7 & 12,8 \\
\hline \multirow{2}{*}{$\mathrm{O}(1 \mathrm{~s})$} & 1 & 531,6 & 49,6 & 531,7 & 39,9 & 531,8 & 45,0 & 531,6 & 45,3 & 531,7 & 43,6 \\
\hline & 2 & 533,3 & 50,4 & 533,3 & 60,1 & 533,1 & 55,0 & 533,0 & 54,7 & 533,0 & 56,4 \\
\hline
\end{tabular}

razão encontrada foi igual à teórica. Idêntica análise foi efetuada para as amostras de PETSpc. Como exemplo, nas figuras 2(c) e 2(d) podem ser visualizados os fotopicos resolvidos do C (1s) e O (1s), respectivamente, para as amostras PETSpc30. Os valores de energia de ligação e concentração atômica relativa das espécies de carbono e oxigênio no PETpc e PETSpc calculadas a partir da resolução dos fotopicos de C (1s) e O (1s) são mostrados na Tabela 1

A partir da análise da Tabela 1, verifica-se que há um aumento relativo da porcentagem do oxigênio éter (fotopico em 533,3 eV) e da porcentagem do carbono éter (fotopico em $286,2 \mathrm{eV}$ ), que é um carbono de oxidação intermediária, nas amostras submetidas à reação com ácido sulfúrico em relação ao material não modificado. Estes aumentos se relacionam com a quebra de cadeias do material, através da hidrólise ácida dos grupamentos éster, conforme a reação se processa.

Através da análise dos espectros obtidos por FTIR-PAS (Figuras 2a) do PET pós-consumo e do material modificado 

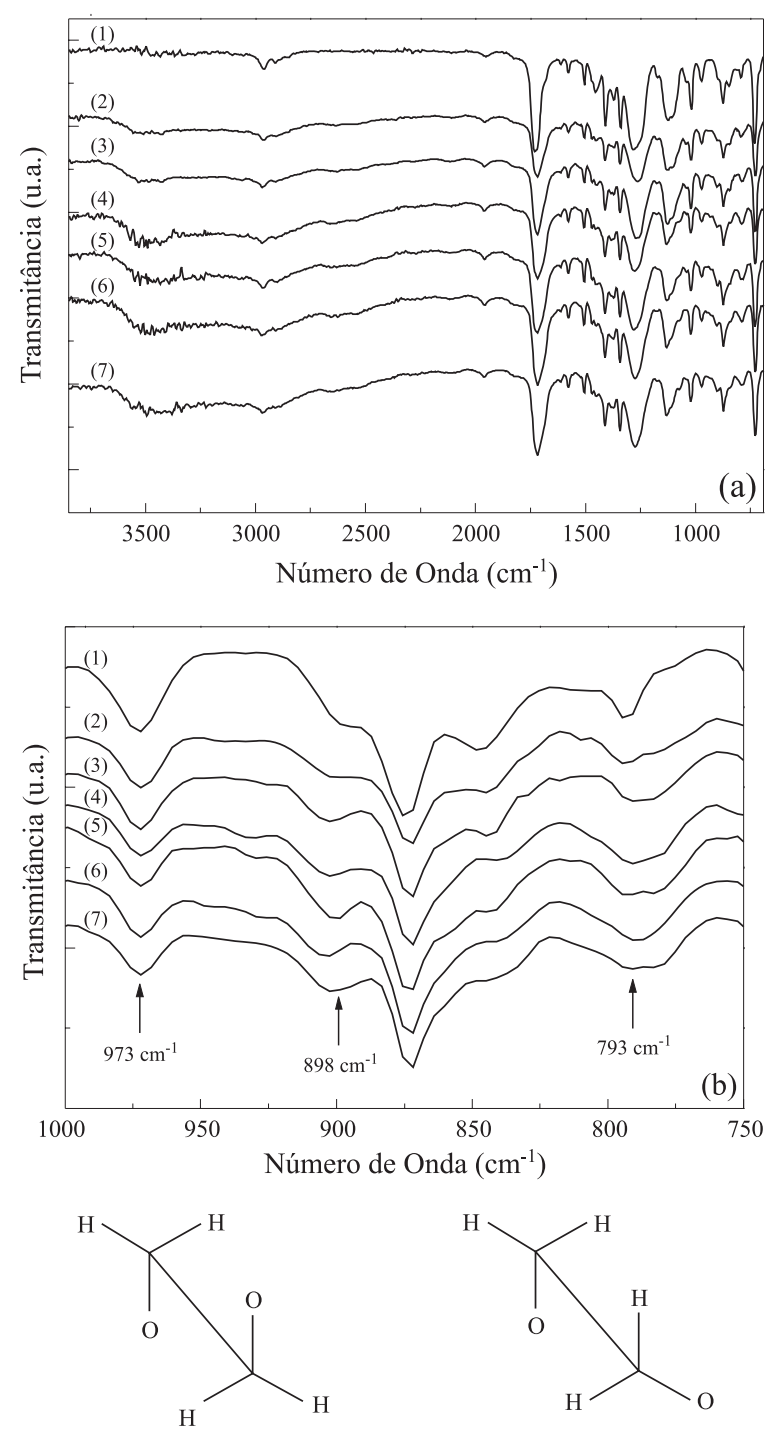

Forma Trans

Forma Gauche

Figura 2. (a) Espectros de FTIR para amostras de PETpc (1); PETSpc10 (2); PETSpc20 (3); PETSpc30 (4); PETSpc60 (5); PETSpc90 (6) e PETSpc120 (7). (b) Região de 750 a $1000 \mathrm{~cm}^{-1}$ ampliada.

foi observado a diminuição da intensidade dos picos referente ao grupamento éster: estiramento assimétrico COC (em $\left.1268 \mathrm{~cm}^{-1}\right)$, estiramento simétrico COC $\left(\mathrm{em} 1127 \mathrm{~cm}^{-1}\right)$. Também verificou-se o alargamento da banda em $1720 \mathrm{~cm}^{-1}$ referente ao grupamento carbonila, que para ésteres está na região de $1725-1730 \mathrm{~cm}^{-1}$ e para ácidos carboxílicos, 1710$1660 \mathrm{~cm}^{-1}$, o que implica um aumento na quantidade de carbonila referente a ácido carboxílico, além do aparecimento da banda larga em aproximadamente $3500 \mathrm{~cm}^{-1}$, referente a grupos hidroxilas.

A figura 2 apresenta a região de 750 a $1000 \mathrm{~cm}^{-1}$ ampliada, onde se podem observar as bandas relacionadas às conformações trans $\left(973 \mathrm{~cm}^{-1}\right)$, gauche $\left(898 \mathrm{~cm}^{-1}\right)$ e a banda de referência interna em $793 \mathrm{~cm}^{-1}$. As intensidades destas bandas foram relacionas com a intensidade da banda em $793 \mathrm{~cm}^{-1}$ obtendo-se razões relativas da conformação gauche $\left(\mathrm{R}_{\mathrm{G}}=\right.$ $\left.\mathrm{A}_{898} / \mathrm{A}_{793}\right)$ e trans $\left(\mathrm{R}_{\mathrm{T}}=\mathrm{A}_{973} / \mathrm{A}_{793}\right)^{[11]}$ e estão apresentadas na

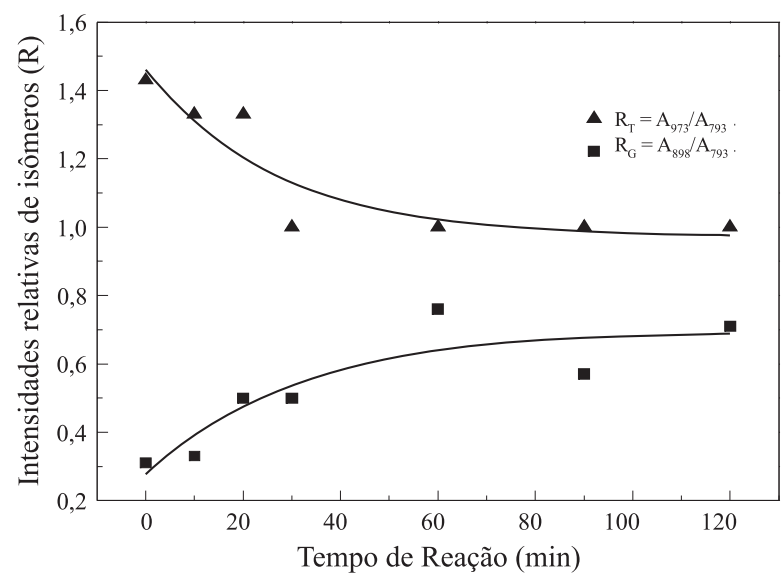

Figura 3. Intensidade relativa dos isômeros trans $\left(\mathrm{R}_{\mathrm{T}}=\mathrm{A}_{973} / \mathrm{A}_{793}\right)$ e gauche $\left(\mathrm{R}_{\mathrm{G}}=\mathrm{A}_{898} / \mathrm{A}_{793}\right)$ para amostras de PETpc em função do tempo de reação com ácido sulfúrico.

Figura 3. Entre 0 e 60 minutos pode-se observar o aumento da intensidade relativa da conformação gauche, característica da região amorfa, e a diminuição da intensidade relativa da conformação trans, que pode estar presente tanto na região cristalina (isômero trans cristalino, $\mathrm{T}^{\mathrm{c}}$ ) como na região amorfa (isômero trans amorfo, $\mathrm{T}^{\mathrm{a}}$ ), indicando que no processo de obtenção do material o isômero trans é favorecido com o aumento do tempo de reação. Para tempos de reação maiores que 60 min a intensidade relativa das bandas referentes às conformações trans $\mathrm{e}$ gauche não apresenta variação significativa.

$\mathrm{Na}$ figura 4 podem ser visualizadas as curvas de DSC para amostras de PETpc e de PETSpc submetidas a diferentes tempos de reação com acido sulfúrico. Pela análise das curvas de DSC verifica-se uma diminuição da temperatura de fusão e o alargamento da endoterma para todos os tempos de reação. Para tempos de reação a partir de 60 minutos observa-se, com maior nitidez, o desdobramento da endoterma de fusão e seu deslocamento para temperaturas inferiores. Têm sido propostos vários modelos ${ }^{[12-14]}$ para explicar a presença de múltiplas endotermas em experimentos de DSC para polímeros. Entre esses modelos estão: (a) modelo de fusão/recristalização; (b) modelo de lamelas de diferentes espessuras e (c) modelo de envelhecimento físico. Para a discussão qualitativa da presença de múltiplas endotermas e do seu deslocamento para temperaturas inferiores, como observado nos experimentos de DSC, considerou-se a equação de Thomson-Gibbs ${ }^{[15]}$,

$$
T m=T^{o} m\left(1-\frac{2 \sigma_{e}}{\Delta H^{o} l_{c}}\right)
$$

onde $\mathrm{T}^{\circ} \mathrm{m}$ e Tm são as temperaturas de fusão termodinâmica e a temperatura de fusão observadas, respectivamente, $l_{\mathrm{c}}$ é a espessura média das lamelas cristalinas, $\sigma_{e}$ é a energia livre de superfície da lamela cristalina e $\Delta \mathrm{H}^{\circ}$ é a entalpia de fusão de um cristal perfeito. $\mathrm{O}$ aumento do tempo de reação favorece a formação de estruturas que se fundem em temperaturas menores (aproximadamente $135^{\circ} \mathrm{C}$ e $181{ }^{\circ} \mathrm{C}$ - Figura 4PETSpc120). A diminuição da temperatura de fusão, de acordo com a equação (1), está relacionada com o tamanho das 


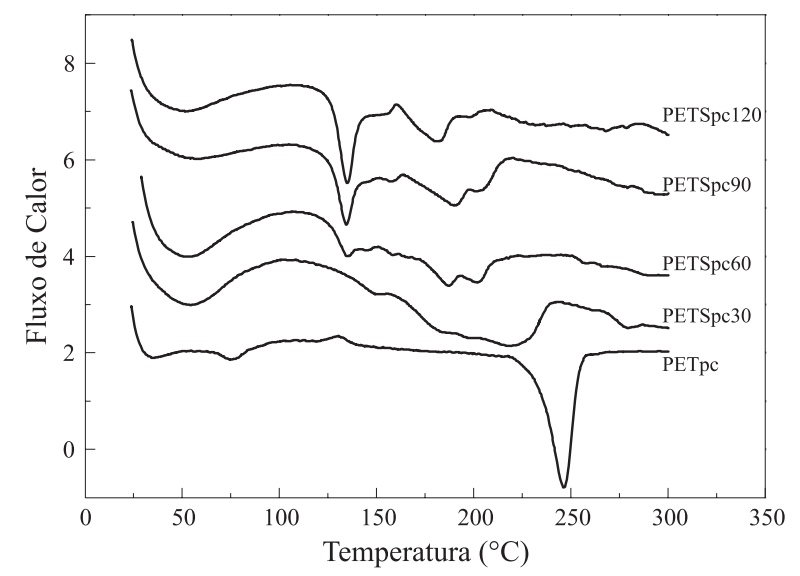

Figura 4. Termogramas de DSC para amostras de PETpc e PETSpc quimicamente modificado por diferentes tempos de reação em ácido sulfúrico.

lamelas cristalinas. Quanto menor as lamelas do cristal, maior o abaixamento da temperatura de fusão do material. A temperatura de fusão termodinâmica determinada, $\mathrm{T}^{\circ} \mathrm{m}$, foi $293^{\circ} \mathrm{C}$. Além do abaixamento da temperatura de fusão, pode-se observar nas curvas de DSC das amostras PETSpc (Figura 4) a presença de mais de uma endoterma, indicando a presença de estruturas que apresentam lamelas de diferentes tamanhos.

A intensidade relativa da banda relacionada ao isômero trans $\left(973 \mathrm{~cm}^{-1}\right)$, em que é detectada a fração total de isômeros trans $\left(\mathrm{T}^{\mathrm{a}}+\mathrm{T}^{\mathrm{c}}\right)$, não apresenta variação a partir de 60 minutos de reação. Portanto a alteração na estrutura do material, detectada pelo deslocamento das endotermas de fusão para temperaturas inferiores nos gráficos de DSC, para todos os tempos de reação, indica que o ordenamento do material está relacionado com estruturas de tamanho menor. A correlação destes dados, a partir de 60 minutos de reação, permite inferir que a formação de estruturas de tamanhos menores, que se fundem em diferentes temperaturas, pode ser relacionada com alteração das quantidades relativas dos isômeros $\mathrm{T}^{\mathrm{a}} \mathrm{e} \mathrm{T}^{\mathrm{c}}$. Esta alteração se deve, possivelmente, à presença de uma maior quantidade de isômero $\mathrm{T}^{\mathrm{a}}$, já que estas estruturas se fundem em temperaturas menores e em um intervalo mais amplo de temperatura devendo, portanto possuir um menor ordenamento ${ }^{[11]}$.

Na Tabela 2 estão apresentados os dados da capacidade de troca iônica do PETpc e do PETSpc em função do tempo de reação. Os valores apresentados são médias obtidas de triplicatas. Como pode ser verificado, o tempo de reação não

Tabela 2. Valores de capacidade de troca iônica para amostras de PETpc e PETSpc

\begin{tabular}{cc}
\hline Amostra & Capacidade de troca $(\mathbf{m e q} / \mathrm{g}) *$ \\
\hline PETpc & $0,28 \pm 0,10$ \\
PETSpc30 & $7,76 \pm 0,12$ \\
PETSpc60 & $8,10 \pm 0,30$ \\
PETSpc90 & $8,13 \pm 0,63$ \\
PETSpc120 & $8,05 \pm 0,07$
\end{tabular}

*Valores determinados por titulação em quantidade de meq em relação ao peso seco da resina usado na determinação. influi significativamente na capacidade de troca iônica do material. A capacidade de troca iônica, determinada por titulação, para o PETSpc é comparável com a de materiais comerciais. Resinas trocadoras catiônicas comercialmente disponíveis (Dowex 50X-8, SP Sephadex C-25, Duolite C225), apresentam capacidade de troca iônica entre valores de 4,5 a $10 \mathrm{meq} / \mathrm{g}$ de resina seca ${ }^{[16]}$.

A porcentagem de corante Remazol vermelho RB retida por amostras de PETSpc30 foi determinada em função da razão de massa de PETSpc30/volume de solução. Os valores encontrados foram de $22,6 \pm 0,7 ; 87,2 \pm 0,5 ; 86,9 \pm 1,2 \mathrm{e}$ $83,1 \pm 0,9$, para razões de $2,5 \% ; 5 \% ; 10 \%$ e $15 \%$, respectivamente. A partir da razão de 5\% massa de PETSpc30/volume de solução a percentagem de adsorção de corante não é influenciada pela quantidade de adsorvente. A isoterma de adsorção do corante Remazol Vermelho RB foi determinada para a amostra PETSpc30, utilizando-se a razão massa de PETSpc30/ volume de solução de 5\% e está apresentada na Figura 5a. Os dados experimentais ajustaram-se a equação de Langmuir, que pode ser sumarizado pela equação 2 ,

$$
q=\frac{q_{\mathrm{m}} \mathrm{C}}{\mathrm{K}+\mathrm{C}}
$$

onde q é a quantidade de corante adsorvida por massa de adsorvente, $\mathrm{C}$ a concentração de equilíbrio de corante na
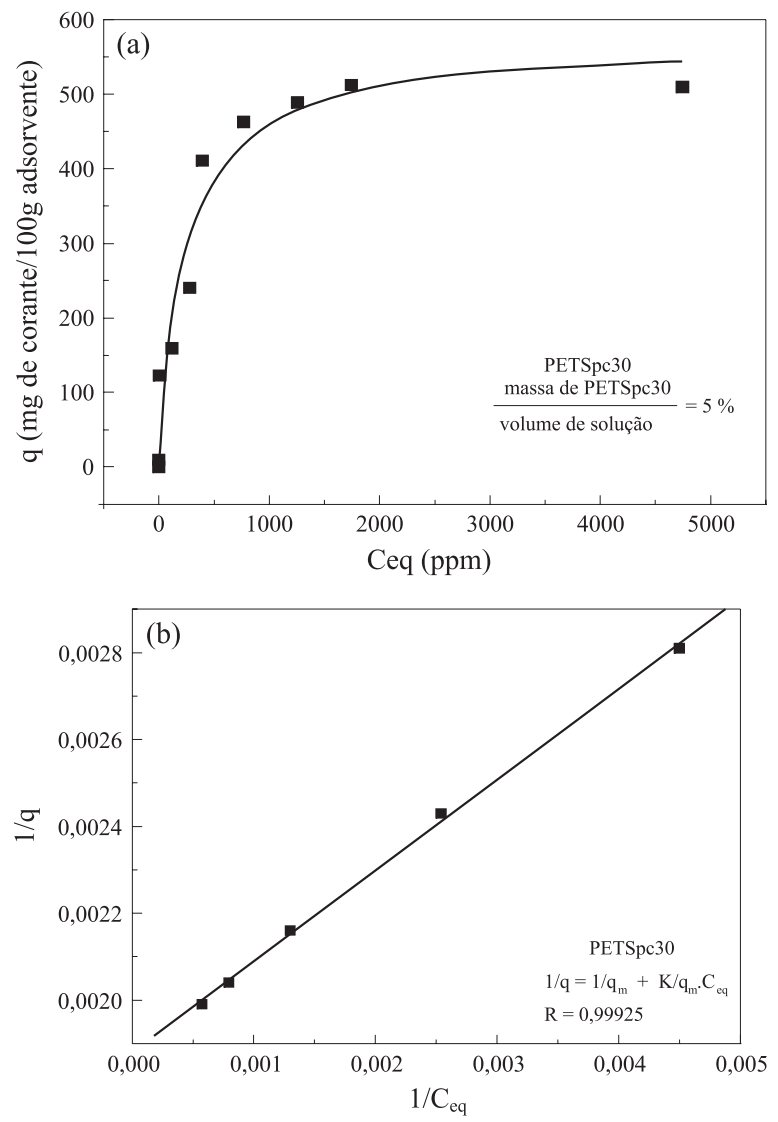

Figura 5. (a) Isoterma de adsorção do corante Remazol Vermelho RB para amostra PETSpc30. (b) Recíproco da capacidade de adsorção em função do recíproco da concentração de equilíbrio para adsorção de corante Remazol Vermelho RB em PETSpc30. 
solução, K é o parâmetro de afinidade entre o adsorvente e o adsorvato e qm a capacidade máxima de adsorção do adsorvente ${ }^{[17]}$, com valores de $\mathrm{K}=111,18 \mathrm{ppm}$ e qm $=531,91$ $\mathrm{mg}$ de corante $/ 100 \mathrm{~g}$ de adsorvente. Para a determinação dos valores de $\mathrm{K}$ e de qm foi feito um gráfico de $1 / \mathrm{q}$ em função de 1/C e uma reta com coeficiente de correlação(R) igual a 0,99925 foi obtida (Figura 5b).

\section{Conclusões}

A correlação dos dados de FTIR-PAS e XPS permite verificar a ocorrência do processo de hidrólise do material durante a modificação química em ácido sulfúrico, nas condições do experimento. A correlação entre os dados de DSC e FTIRPAS para amostras quimicamente modificadas entre zero e 60 minutos indica que houve modificação na estrutura do material. Isto foi observado pela variação das intensidades relativas das bandas associadas aos isômeros trans $\left(898 \mathrm{~cm}^{-1}\right)$ e gauche $\left(973 \mathrm{~cm}^{-1}\right)$ e pelo alargamento e deslocamento das endotermas de fusão para temperaturas inferiores. A partir de 60 minutos de reação há o aparecimento de mais de uma endoterma de fusão nos termogramas de DSC, possivelmente devido a presença de estruturas formadas pelo isômero trans presente na região amorfa $\left(\mathrm{T}^{\mathrm{a}}\right)$.

A hidrólise de PETpc utilizando ácido sulfúrico como reagente propicia a obtenção de material com boa capacidade de troca iônica, comparável com a de materiais comerciais. O modelo de adsorção de Langmuir, que prevê a adsorção em monocamadas, é compatível com os dados experimentais. A porcentagem de corante retido no PETSpc indica que esse material possui boas características adsorventes podendo vir a ser utilizado para tratamento de efluente têxtil, agregando valor ao material reciclado.

\section{Referências Bibliográficas}

1. Encyclopedia of Polymer Science and Technology. Interscience Publisher, v. 12, p. 193 (1987).

2. Shashin, M. M. - Polymer Testing, v. 14, p. 243 (1995).

3. Brandrup, J. \& Immergut, E. H. - Polymer Handbook, 3rd ed., New York: Wiley (1989).
4. Modern Plastics Encyclopedia Handbook, McGraw-Hill, Inc. (1994).

5. Ward, S.; Jones, K. M. \& Marbrow, R. A. - "Recycling of Polyester: An Industry Perspective”, in: Chemical Aspects of Plastics Recycling. Hoyle, W. and Karsa, D.R. The Royal Society of Chemistry, Cambridge, U.K: 79 (1997).

6. Yang, Y.; Liu, Y.; Xiang, H.; Xu, Y. \& Li, Y. - Polymer Degradation and Stability, v. 75, p. 185 (2002).

7. Mansour, S. H. \& Ikladious, N. E. - Polymer Testing, v. 21, p. 497 (2002).

8. Lin, S. B. \& Koenig, J. L. - Journal. of Polymer Science., Polymer Physics ed., vol. 20, p. 2277 (1982).

9. Helfferich, F. - "Ion Exchange", McGraw-Hill Book Company, Inc., New York (1962).

10. Koroioshi, E. K.; Bonan, A. A.; Andrade, C. B.; Silva, A. F.; Santos, W. L. F. \& Silva, C. F. - Acta Scientarium, v. 22 (5), p. 1185 (2000).

11. Ruvolo-Filho, A. \& Carvalho, G. M. - Journal of Macromolecular Science Physics, B38(3), p.305 (1999).

12. Verma, R. K. \& Hsiao, B. S.. TRIP. vol.4, n.9 (1996).

13. Sauer, B. B.; Kampert, W. G.; Blanchad, E. N. ; Threefoot, S. A. \& Hsiao, B. S. - Polymer, vol. 41, p. 1099 (2000).

14. Wang, Z.-G.; Hsiao, B. S.; Sauer, B. B., \& Kampert, W. G. - Polymer, 40, p. 4615 (1999).

15. Groeninckx G.; Reynaers, H.; Berghmans, H. \& Smets, G. - Journal of Polymer Science: Polymer Physics Edition, vol 18, 1311 - 1324, (1980).

16. Collins, C. H.; Braga, G. L. \& Bonato, P. S. - "Introdução a métodos cromatográficos." UNICAMP, Campinas (1997).

17. Klug, M.; Sanches, M. N. M.; Laranjeira, M. C. M.; Fávere, V. T. \& Rodrigues, C. A. - Química Nova, v. 21 (4), p. 410 (1998).

Enviado: 03/03/04

Reenviado: 08/11/04

Aprovado: 16/11/04 